Supplement of Biogeosciences Discuss., 12, 1907-1973, 2015

http://www.biogeosciences-discuss.net/12/1907/2015/

doi:10.5194/bgd-12-1907-2015-supplement

(C) Author(s) 2015. CC Attribution 3.0 License.

(c) (i)

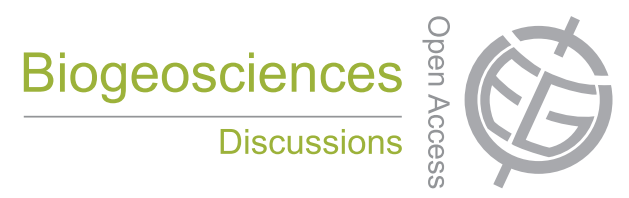

Supplement of

\title{
WETCHIMP-WSL: intercomparison of wetland methane emissions models over West Siberia
}

\section{T. J. Bohn et al.}

Correspondence to: T. J. Bohn (theodore.bohn@asu.edu) 
Supplement of Biogeosciences Discuss., 12, 1907-1973, 2015

http://www.biogeosciences-discuss.net/12/1907/2015/

doi:10.5194/bgd-12-1907-2015-supplement

(C) Author(s) 2015. CC Attribution 3.0 License.

(c) (i)

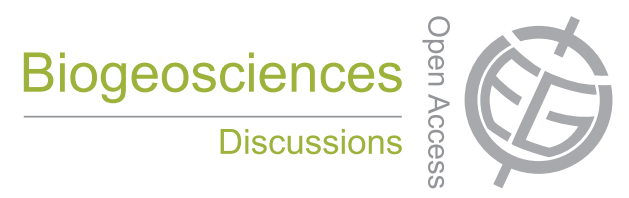

Supplement of

\section{WETCHIMP-WSL: intercomparison of wetland methane emissions models over West Siberia}

\section{T. J. Bohn et al.}

Correspondence to: T. J. Bohn (theodore.bohn@asu.edu) 
Table S1. Estimates of annual $\mathrm{CH}_{4}$ emissions and June-July-August $\mathrm{CH}_{4}$ emissions, contributing areas, and $\mathrm{CH}_{4}$ intensities from models, observational flux-area estimates, and inversions, over the entire WSL and its Southern $\left(<61^{\circ} \mathrm{N}\right)$ and Northern halves, for the period $1993-2004$.

\begin{tabular}{|c|c|c|c|c|c|c|c|c|c|c|c|c|}
\hline \multirow[t]{2}{*}{ Estimate } & \multicolumn{3}{|c|}{$\begin{array}{l}\text { Average Annual } \mathrm{CH}_{4} \text { Emissions } \\
\left(\mathrm{Tg} \mathrm{CH}_{4} \mathrm{y}^{-1}\right)\end{array}$} & \multicolumn{3}{|c|}{$\begin{array}{l}\text { Average June-July-August } \mathrm{CH}_{4}(\mathrm{Tg} \\
\left.\mathrm{CH}_{4} \text { month }^{-1}\right)\end{array}$} & \multicolumn{3}{|c|}{$\begin{array}{l}\text { Average June-July-August Contributing } \\
\text { Area }\left(10^{3} \mathbf{k m}^{2}\right)\end{array}$} & \multicolumn{3}{|c|}{$\begin{array}{l}\text { Average June-July-August } \mathrm{CH}_{4} \text { Intensity } \\
\left(\mathrm{g} \mathrm{CH}_{4} \mathrm{~m}^{-2} \mathrm{mon}^{-1}\right)\end{array}$} \\
\hline & WSL & $\mathbf{S}$ & $\mathbf{N}$ & WSL & $\mathbf{S}$ & $\mathbf{N}$ & WSL & $\mathbf{S}$ & $\mathbf{N}$ & WSL & $\mathbf{S}$ & $\mathbf{N}$ \\
\hline \multicolumn{13}{|l|}{ Models } \\
\hline CLM4Me & 5.77 & 1.10 & 4.67 & 1.32 & 0.21 & 1.11 & 390 & 64 & 325 & 3.39 & 3.22 & 3.42 \\
\hline DLEM & 3.72 & 1.23 & 2.49 & 0.75 & 0.19 & 0.56 & 368 & 92 & 276 & 2.03 & 2.05 & 2.03 \\
\hline DLEM2 & 3.28 & 0.43 & 2.85 & 0.74 & 0.07 & 0.67 & 398 & 49 & 350 & 1.85 & 1.34 & 1.92 \\
\hline IAP-RAS ${ }^{\mathrm{a}}$ & 11.19 & 9.29 & 1.90 & 3.69 & 3.07 & 0.62 & 461 & 321 & 140 & 8.01 & 9.57 & 4.44 \\
\hline LPJ-Bern & 5.49 & 0.99 & 4.50 & 1.03 & 0.14 & 0.89 & 635 & 112 & 523 & 1.63 & 1.28 & 1.70 \\
\hline LPJ-MPI & 4.77 & 2.72 & 2.05 & 1.16 & 0.62 & 0.54 & 456 & 166 & 290 & 2.54 & 3.70 & 1.88 \\
\hline LPJ-WHyMe & 7.87 & 1.26 & 6.61 & 1.75 & 0.26 & 1.49 & 405 & 51 & 354 & 4.31 & 5.09 & 4.21 \\
\hline LPJ-WSL & 4.01 & 0.84 & 3.17 & 1.06 & 0.15 & 0.91 & 233 & 30 & 202 & 4.55 & 5.02 & 4.48 \\
\hline LPX-BERN (N) & 3.07 & 1.90 & 1.17 & 0.67 & 0.39 & 0.28 & 710 & 341 & 369 & 0.94 & 1.15 & 0.76 \\
\hline \multicolumn{13}{|l|}{ LPX-BERN } \\
\hline (DYPTOP-N) & 2.42 & 1.36 & 1.06 & 0.49 & 0.27 & 0.22 & 563 & 223 & 340 & 0.88 & 1.21 & 0.66 \\
\hline ORCHIDEE & 6.47 & 2.52 & 3.96 & 1.72 & 0.53 & 1.19 & 303 & 51 & 252 & 5.69 & 10.50 & 4.73 \\
\hline SDGVM & 5.69 & 3.93 & 1.76 & 1.57 & 1.05 & 0.52 & 1165 & 549 & 616 & 1.35 & 1.92 & 0.84 \\
\hline UW-VIC (GIEMS) & 5.05 & 2.07 & 2.98 & 1.03 & 0.52 & 0.51 & 681 & 292 & 389 & 1.51 & 1.77 & 1.32 \\
\hline \multirow{2}{*}{\multicolumn{13}{|c|}{ VIC-TEM- }} \\
\hline & & & & & & & & & & & & \\
\hline TOPMODEL & 8.74 & 4.72 & 4.02 & 2.46 & 1.31 & 1.15 & 547 & 238 & 309 & 4.49 & 5.51 & 3.71 \\
\hline VISIT (GLWD) & 4.62 & 1.66 & 2.96 & 0.76 & 0.22 & 0.54 & 590 & 252 & 337 & 1.29 & 0.88 & 1.59 \\
\hline VISIT (SHENG) & 5.08 & 2.24 & 2.84 & 0.81 & 0.30 & 0.51 & 675 & 336 & 338 & 1.20 & 0.88 & 1.52 \\
\hline Mean & 5.34 & 2.38 & 2.96 & 1.29 & 0.58 & 0.70 & 545 & 203 & 341 & 2.76 & 3.36 & 2.35 \\
\hline Std. Dev. & 2.24 & 2.09 & 1.46 & 0.77 & 0.70 & 0.35 & 209 & 140 & 106 & 1.96 & 2.85 & 1.43 \\
\hline Std. Err. & 0.54 & 0.51 & 0.35 & 0.19 & 0.17 & 0.09 & 51 & 34 & 26 & 0.47 & 0.69 & 0.35 \\
\hline \multicolumn{13}{|l|}{ Observations } \\
\hline Glagolev2011 & 3.92 & 2.82 & 1.09 & 0.96 & 0.67 & 0.30 & 679 & 336 & 343 & 1.42 & 1.99 & 0.87 \\
\hline \multicolumn{13}{|l|}{ Inversions } \\
\hline Kim2011 & 3.08 & 2.42 & 0.66 & 0.75 & 0.58 & 0.18 & 679 & 336 & 343 & 1.11 & 1.72 & 0.52 \\
\hline Bloom2010 & 3.71 & 2.22 & 1.49 & $\mathrm{n} / \mathrm{a}$ & $\mathrm{n} / \mathrm{a}$ & $\mathrm{n} / \mathrm{a}$ & $\mathrm{n} / \mathrm{a}$ & $\mathrm{n} / \mathrm{a}$ & $\mathrm{n} / \mathrm{a}$ & $\mathrm{n} / \mathrm{a}$ & $\mathrm{n} / \mathrm{a}$ & $\mathrm{n} / \mathrm{a}$ \\
\hline Winderlich2012 & 9.80 & 3.43 & 6.37 & 1.58 & 0.56 & 1.02 & $\mathrm{n} / \mathrm{a}$ & $\mathrm{n} / \mathrm{a}$ & $\mathrm{n} / \mathrm{a}$ & $\mathrm{n} / \mathrm{a}$ & $\mathrm{n} / \mathrm{a}$ & $\mathrm{n} / \mathrm{a}$ \\
\hline Bousquet2011K & 6.98 & 2.30 & 4.68 & 1.47 & 0.49 & 0.98 & $\mathrm{n} / \mathrm{a}$ & $\mathrm{n} / \mathrm{a}$ & $\mathrm{n} / \mathrm{a}$ & $\mathrm{n} / \mathrm{a}$ & $\mathrm{n} / \mathrm{a}$ & $\mathrm{n} / \mathrm{a}$ \\
\hline Bousquet2011R & 6.73 & 3.37 & 3.36 & 1.63 & 0.78 & 0.85 & $\mathrm{n} / \mathrm{a}$ & $\mathrm{n} / \mathrm{a}$ & $\mathrm{n} / \mathrm{a}$ & $\mathrm{n} / \mathrm{a}$ & $\mathrm{n} / \mathrm{a}$ & $\mathrm{n} / \mathrm{a}$ \\
\hline Mean & 6.06 & 2.75 & 3.31 & 1.36 & 0.60 & 0.76 & $\mathrm{n} / \mathrm{a}$ & $\mathrm{n} / \mathrm{a}$ & $\mathrm{n} / \mathrm{a}$ & $\mathrm{n} / \mathrm{a}$ & $\mathrm{n} / \mathrm{a}$ & $\mathrm{n} / \mathrm{a}$ \\
\hline Std. Dev. & 2.72 & 0.60 & 2.32 & 0.41 & 0.12 & 0.39 & $\mathrm{n} / \mathrm{a}$ & $\mathrm{n} / \mathrm{a}$ & $\mathrm{n} / \mathrm{a}$ & $\mathrm{n} / \mathrm{a}$ & $\mathrm{n} / \mathrm{a}$ & $\mathrm{n} / \mathrm{a}$ \\
\hline Std. Err. & 1.22 & 0.27 & 1.04 & 0.20 & 0.06 & 0.20 & $\mathrm{n} / \mathrm{a}$ & $\mathrm{n} / \mathrm{a}$ & $\mathrm{n} / \mathrm{a}$ & $\mathrm{n} / \mathrm{a}$ & $\mathrm{n} / \mathrm{a}$ & $\mathrm{n} / \mathrm{a}$ \\
\hline
\end{tabular}

${ }^{\mathrm{a}}$ For IAP-RAS, JJA emissions were set equal to the annual total

Table S2. Temporal correlations between $\mathrm{JJA} \mathrm{CH}_{4}$ emissions and environmental drivers, 1993-2004

\begin{tabular}{|c|c|c|c|c|c|c|c|c|c|c|c|c|}
\hline Model & WSL & & & & $\mathbf{S}$ & & & & $\mathbf{N}$ & & & \\
\hline & $\begin{array}{l}\text { CRU } \\
\text { P } \\
\end{array}$ & $\begin{array}{l}\text { CRU } \\
\mathbf{T}_{\text {air }} \\
\end{array}$ & $\begin{array}{l}\text { GIEMS } \\
\text { F }_{\text {inumd }}{ }^{2} \\
\end{array}$ & $\begin{array}{l}\text { SWAMPS } \\
\text { F }_{\text {inund }}{ }^{2} \\
\end{array}$ & CRU P & $\begin{array}{l}\text { CRU } \\
\mathbf{T}_{\text {air }} \\
\end{array}$ & $\begin{array}{l}\text { GIEMS } \\
\text { F }_{\text {inund }}{ }^{2} \\
\end{array}$ & $\begin{array}{l}\text { SWAMPS } \\
\text { F }_{\text {inund }}{ }^{2} \\
\end{array}$ & $\begin{array}{l}\text { CRU } \\
\mathbf{P} \\
\end{array}$ & $\begin{array}{l}\text { CRU } \\
\mathbf{T}_{\text {air }} \\
\end{array}$ & $\begin{array}{l}\text { GIEMS } \\
\text { F }_{\text {inumd }}{ }^{2} \\
\end{array}$ & $\begin{array}{l}\text { SWAMPS } \\
\text { F }_{\text {inund }}{ }^{2} \\
\end{array}$ \\
\hline CLM4Me & -0.24 & 0.58 & 0.32 & 0.29 & 0.01 & 0.66 & 0.24 & 0.40 & -0.05 & 0.41 & 0.28 & 0.23 \\
\hline
\end{tabular}




\begin{tabular}{|c|c|c|c|c|c|c|c|c|c|c|c|c|}
\hline DLEM & 0.46 & 0.18 & 0.87 & 0.48 & 0.54 & 0.09 & 0.81 & 0.69 & 0.35 & 0.23 & 0.84 & 0.40 \\
\hline DLEM2 & 0.32 & 0.40 & 0.64 & 0.33 & 0.33 & 0.23 & 0.83 & 0.63 & 0.02 & 0.42 & 0.60 & 0.25 \\
\hline IAP-RAS ${ }^{b}$ & 0.03 & 0.65 & -0.22 & 0.36 & -0.26 & 0.74 & -0.56 & 0.07 & 0.23 & 0.73 & -0.12 & 0.28 \\
\hline LPJ-Bern & 0.62 & 0.23 & 0.15 & 0.45 & 0.74 & -0.19 & 0.50 & 0.65 & 0.38 & 0.40 & 0.07 & 0.41 \\
\hline LPJ-MPI & 0.36 & 0.16 & 0.63 & 0.30 & 0.45 & 0.24 & 0.49 & 0.64 & 0.22 & -0.04 & 0.71 & 0.20 \\
\hline LPJ-WHyMe & 0.69 & -0.07 & 0.35 & 0.51 & 0.46 & -0.32 & 0.61 & 0.71 & 0.73 & 0.06 & 0.27 & 0.41 \\
\hline LPJ-WSL & 0.55 & 0.11 & 0.93 & 0.63 & 0.30 & 0.07 & 0.95 & 0.79 & 0.47 & 0.17 & 0.91 & 0.57 \\
\hline LPX-BERN & -0.12 & 0.88 & -0.28 & 0.13 & -0.62 & 0.75 & -0.40 & -0.40 & 0.15 & 0.92 & -0.05 & 0.36 \\
\hline LPX-BERN (DYPTOP) & -0.14 & 0.93 & -0.02 & 0.30 & -0.04 & 0.77 & 0.18 & 0.15 & -0.18 & 0.95 & -0.09 & 0.35 \\
\hline LPX-BERN (N) & -0.27 & 0.94 & -0.33 & -0.03 & -0.54 & 0.86 & -0.41 & -0.46 & -0.04 & 0.94 & -0.08 & 0.23 \\
\hline LPX-BERN (DYPTOP-N) & -0.32 & 0.94 & -0.14 & 0.09 & -0.32 & 0.88 & -0.17 & -0.24 & -0.28 & 0.95 & -0.07 & 0.25 \\
\hline ORCHIDEE & -0.09 & 0.79 & 0.05 & 0.18 & -0.04 & 0.75 & 0.13 & 0.21 & 0.16 & 0.66 & -0.05 & 0.18 \\
\hline SDGVM & 0.33 & 0.74 & 0.38 & 0.32 & 0.33 & 0.58 & 0.34 & 0.25 & 0.54 & 0.67 & 0.06 & 0.39 \\
\hline UW-VIC (GIEMS) & -0.34 & 0.10 & -0.07 & -0.57 & -0.31 & 0.43 & 0.02 & -0.20 & -0.32 & -0.11 & -0.11 & -0.64 \\
\hline UW-VIC (SWAMPS) & 0.14 & 0.59 & 0.47 & 0.39 & 0.17 & 0.44 & 0.55 & 0.44 & -0.01 & 0.67 & 0.10 & 0.23 \\
\hline VIC-TEM-TOPMODEL & 0.67 & 0.20 & 0.53 & 0.41 & 0.71 & 0.08 & 0.42 & 0.54 & 0.43 & 0.29 & 0.46 & 0.47 \\
\hline VISIT (GLWD) & -0.03 & 0.95 & 0.03 & 0.16 & 0.01 & 0.90 & -0.02 & -0.07 & -0.14 & 0.96 & 0.08 & 0.26 \\
\hline VISIT (Sheng) & -0.08 & 0.95 & 0.00 & 0.11 & -0.01 & 0.91 & -0.02 & -0.07 & -0.16 & 0.96 & 0.04 & 0.24 \\
\hline VISIT (GLWD-WH) & 0.05 & 0.80 & 0.07 & 0.23 & -0.08 & 0.84 & 0.12 & 0.12 & 0.15 & 0.76 & 0.07 & 0.33 \\
\hline VISIT (Sheng-WH) & -0.02 & 0.79 & 0.06 & 0.17 & -0.08 & 0.84 & 0.12 & 0.12 & 0.10 & 0.74 & 0.06 & 0.29 \\
\hline Bousquet - Kaplan & 0.53 & 0.24 & 0.37 & 0.26 & 0.52 & 0.21 & 0.44 & 0.48 & 0.39 & 0.25 & 0.32 & 0.21 \\
\hline Bousquet - Ref & 0.46 & -0.02 & 0.59 & 0.30 & 0.40 & -0.02 & 0.60 & 0.63 & 0.38 & -0.03 & 0.57 & 0.20 \\
\hline
\end{tabular}

${ }^{\mathrm{a}} \mathrm{F}_{\text {inund }}=$ fractional inundated area

${ }^{\mathrm{b}}$ For IAP-RAS, correlations are between annual $\mathrm{CH}_{4}$ and annual average values of drivers 